\title{
وسائل الإعلام في تعليمية اللغة العربية
}

\section{Zulfa Aimunah, Zakiyah Isnawati}

E-mail: aimunazulfha@gmail.com

Institut Agama Islam Negeri Kudus

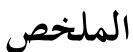

سيكون تعلم اللغة العربية بوسائل الإعلام أكثر إثارة للاهتمام وسهل التعلم، وتذكر،

وفهم، وحفظ، وممارسة الطلاب. لكي يكون تعلم اللغة العربية أكثر فاعلية يجب على المعلمين اختيار الوسائل التي تتناسب مع أهداف ومناهج وطرق تعلم اللغة العربية. يتطلب تطبيق المنهج القائم على الكفاءة في تعلم اللغة العربية وسائل الإعلام في كل عملية لتعلم اللغة العربية. بالإضافة إلى ذلك، يجب على المعلمين اختيار الوسائط المناسبة، لأن هناك العديد من أنواع الوسائط ذات الوظائف المختلفة. بالإضافة إلى ذلك، يجب على المعلمين معرفة العديد من العوامل، بما في ذلك الصعوبات في إعداد وسائل الإعلام أو العوامل التي تدعم تنفيذ وسائل الإعلام نفسها. باختصار، يجب أن يكون المعلمون أكثر إبداعًا في إيجاد وسائل الإعلام وصنعها وإعدادها. الكلمات المفتاحية: الإعلام، التعلم، اللغة العربية. 


\begin{abstract}
Abstrak
Pembelajaran bahasa Arab melalui media akan lebih menarik dan mudah dipelajari, diingat, dipahami, dihafal dan dipraktikkan oleh siswa. Untuk membuat pembelajaran bahasa Arab lebih efektif, para guru harus memilih media yang sesuai dengan tujuan, pendekatan, metode, dan teknik pembelajaran bahasa Arab. Implementasi Kurikulum Berbasis Kompetensi dalam pembelajaran bahasa Arab menuntut media dalam setiap proses pembelajaran bahasa Arab. Selain itu, para guru harus memilih media yang sesuai, karena ada banyak jenis media dengan fungsi yang berbeda. Selain itu, para guru harus mengetahui banyak faktor, termasuk kesulitan dalam menyiapkan media atau faktor yang akan mendukung implementasi media itu sendiri. Singkatnya, para guru harus lebih kreatif dalam mencari, membuat, dan menyiapkan media.
\end{abstract}

Kata kunci: Media, Pembelajaran, Bahasa Arab.

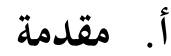

$$
\begin{aligned}
& \text { تعلم اللغة العربية له العائقة الخاصة بأسباب مختلفات منها العقبات و التحدي. } \\
& \text { يوجد العقبات وهو الضعف إلى إتقان اللغات الأجنبية في البحتمع وخاصة من جانب } \\
& \text { السماع (sima) والمحادثة (muhadatsah). يوجد التحدي أيضًا وهو التسهيلات المحدودات } \\
& \text { خاصةً وسائل الإعلام التعليمية. ومع ذلك توجد ايضا عقبة أخرى ، مثل قدرة المعلم } \\
& \text { والدوافع الضعفاء من الطلاب وغيرها. ولذلك يجب إزالة هذه العقبات والتحديات حتى } \\
& \text { يصبح تعلم اللغة العربية في إندونيسيا أكثر كفاءة وفعالية. التعلم الفعال والكفء يؤدي } \\
& \text { إلى جودة الخريجين الذين يتأهلون لللغة العربية. ويجب تحسين تعلم الوسائط في تعليم } \\
& \text { اللغة العربية من حيث النوع وتنوع الاستخدام. سيشكل تعلم اللغة العربية مهارات } \\
& \text { واضحة للطلاب ، وهي مهارات القراءة والكتابة والاستماع والتحدث باللغة العربية. ) } \\
& \text { من ناحية أخرى، يعد يعلم أنّ الوسائط أحد وسائل في تحسين أنشطة التعلم. كل } \\
& \text { نشاط تعليم في الفصل، يريد كل معلم أن تحصل أنشطته نتيجة جيدة بمعنى المؤشرات }
\end{aligned}
$$

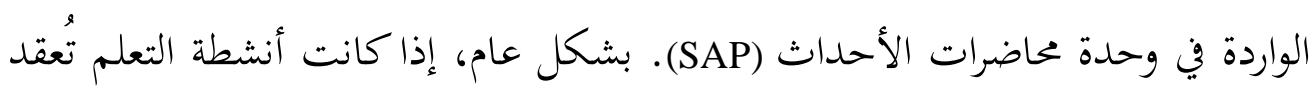

$$
\begin{aligned}
& \text { رسميًا في المدارس، فلن يقصد بأي شيء إلا توجيه التغييرات في كل طالب بطريقة بطدات }
\end{aligned}
$$


مخططة، من حيث المعرفة والمهارات والموقف. تتأثر التفاعلات التي تحدث أثناء عملية

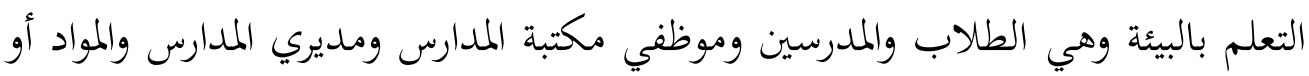
المواد في شكل: الكتب والوحدات النمطية والمنشورات والمحلات وتسجيلات الفيديو والصوت وما شابه ذلك، والعديد من الموارد والمرافق التعليمية مثل: أجهزة الإسقاط العلوية ومسجلات الأشرطة الصوتية ومقاطع الفيديو والإذاعة والتلفزيون وأجهزة الكمبيوتر والمكتبات والمختبرات ومراكز مصادر التعلم وغيرها. Azhar Arsyad, 2007 )

من أجل تحصيل أقصى النتائج يحتاج إلى النظر في مختلف العوامل الداعمة التي

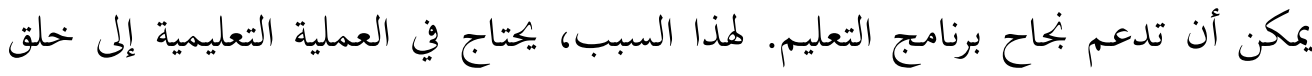

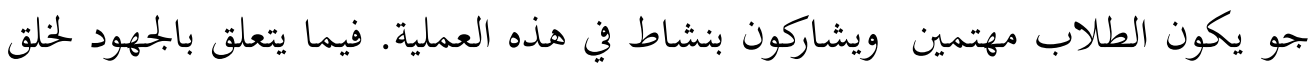
هذا الجمو تعتبر وسائل الإعلام أحد العوامل التي تحدد بنحاح التدريس. يعتمد استخدام

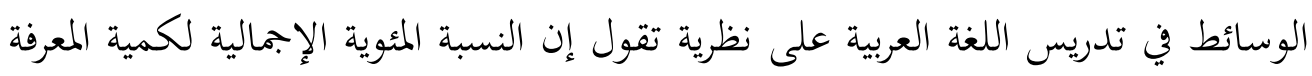

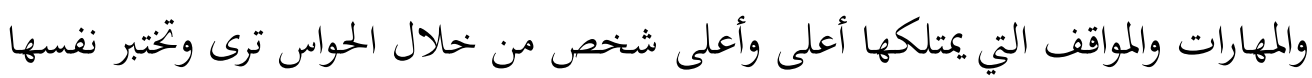
بشكل مباشر، في حين أن البقية من خلال السمع والحواس الأخرى. Soenjoyo Dirjo) Soemarto, 2003, 75) بحيث يمكن استخدام وسائط التعلم بفعالية وكفاءة في تحقيق هدف الإعداد قبل استخدامه وجو الهدوء عند استخدامه. وكذا يجب أن تتكيف وسائل الإعلام المستعملة مع ظروف الطلاب وفقا للمادة التي تم تدريسها. والإعلام المستخدم في تعلم اللغة العربية

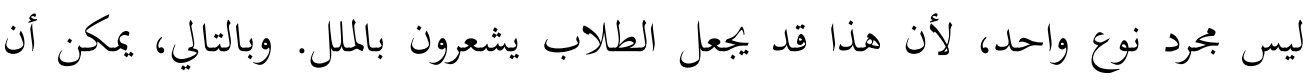

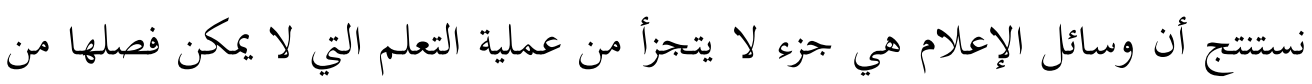

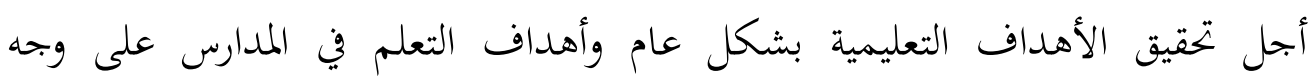
الخصوص. 


\section{ب.مفهوم الوسائل في تعليمية اللغة العربية}

علم أن الوسائل لها معنى خاص. جاء لفظ وسائل الإعلام "media" من اللغة اللاتينية ، هو صيغة الجمع من لفظ "medium" معناه لغة وسيط أو مقدمة. الوسائل هي

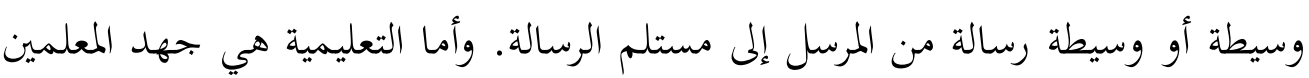
لمساعدة الطلاب على القيام بأنشطة التعلم. (Abdul Hamid, dkk., 2008. 168) وتختلف فرسلف التعريفات لهذه الوسائل وفيما يلي نتعرف لبعض هذه التعاريف :

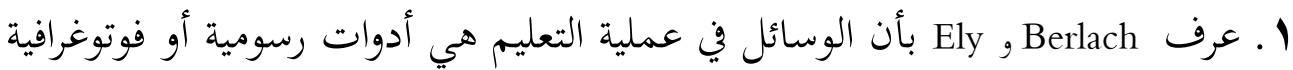
أو إلكترونية لالتقاط المعلومات ومعالجتها وإعادة بنائها وإعادة ترتيب المعلومات

$$
\text { المرئية أو اللفظية. }
$$

r. عرف Heinich و غيره بأن وسائل التعليمية هي وسيط يحمل على رسائل أو معلومات تحدف إلى التعلم أو تحتوي على نوايا تعليمية. r. أما مالك فعرفها بأهما كل ما يمكن استخدامه لتوجيه الرسالة (مواد تعليمية)، بحيث يمكن تحفيز انتباه المتعلم واهتماماته وأفكاره ومشاعره في أنشطة التعلم لتحقيق

$$
\text { أهداف تعليمية معينة. }
$$

ع. وعرفها Briggs Martin بأن وسيلة التعليمية تشتمل على جميع الموارد اللازمة للتواصل مع المتعلم. يمكن أن يكون ذلك في شكل أجهزة وبرامج تستخدم على الأجهزة.). (Abdul Hamid, dkk., 2008 168-169.)

هناك العديد من الخصائص العامة الواردة في تعريف الوسائط على النحو التالي:

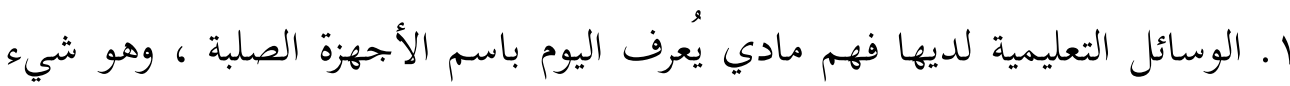
يمكن رؤيته أو سماعه أو لمسه للحواس الخمس. 
r. التعليم الإعلامي لديه فهم غير مادي يعرف بالبربحيات، وهو محتوى الرسائل

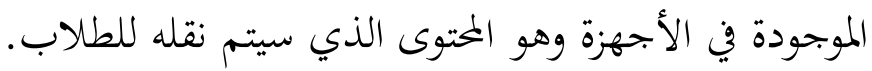

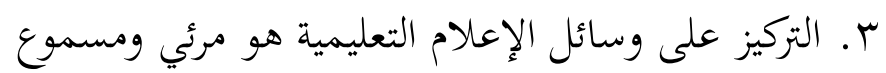

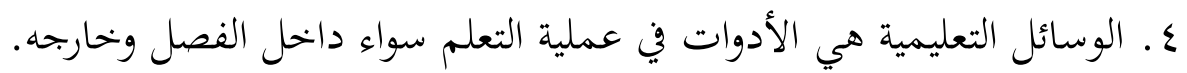
ه. يستخدم الوسائل التعليمية في سياق التواصل والتفاعل بين المعلمين والطلاب في عملية التعلم.

7. يمكن استخدام الوسائل التعليمية بشكل جمعي (على سبيل المثال: الإذاعة والتلفزيون) بحموعات كبيرة وبحموعات صغيرة (مثل الأفلام ومقاطع الفيديو وأوهس)

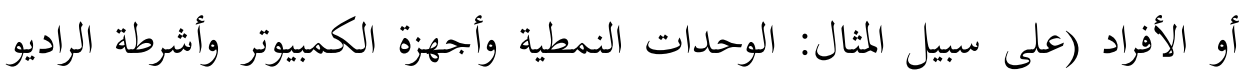

$$
\text { والأشرطة ومسجحلات الفيديو) }
$$

V. المواقف، الإجراءات، التنظيم، الإستراتيجية، والإدارة المتعلقة بتطبيق العلم.

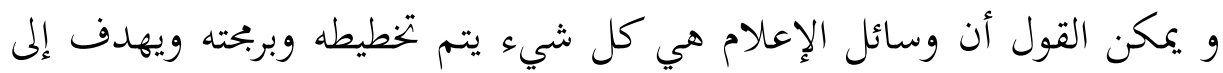

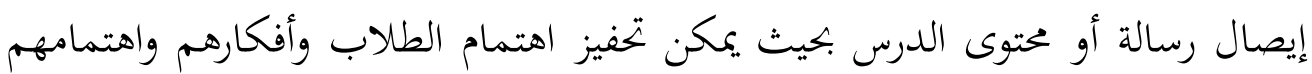
ومشاعرهم وسلوكهم في أنشطة التعليم والتعلم من أجل تحقيق أهداف التعلم المحددة

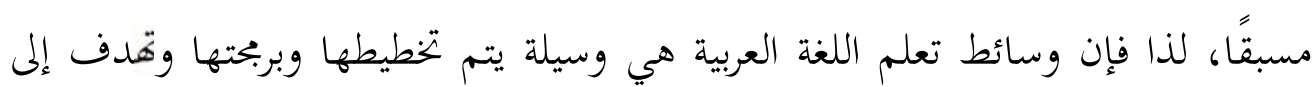
فهم الطلاب للرسائل المادية باللغة العربية وتحقيق أهداف تعلم اللغة العربية.

\section{ج. دور وسائل الإعلام في التعلمية}

اللغة العربية هي لغة تستخدمها الدول العالية حول غري دجلة والفرات، وسهول سيناء وشبه الجزيرة العربية التي لا تزال مستخدمة حتى اليوم.

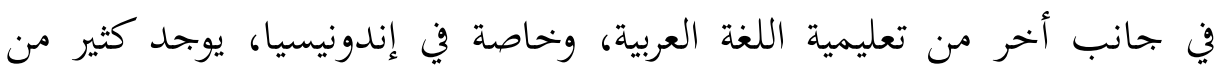
المشكلات التي تنشأ في هذا البحال. من المشكلات أن اللغة العربية هي اللغة الثالثة بعد 
اللغة الأم والإندونيسية بحيث تواجه كثير الصعوبات التي يواجهها المتعلمون. الصعوبات

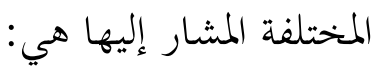
1. نطق العديد من الأصوات / الالفاظ التي تخالف أصوات الإندونيسية. r. رسوم الحروف / الكلمات / الجمل التي تختلف عن اللغة التي يتقنها المتعلم. r. ضبط معاني الكلمات المتنوعة للغاية باللغة العربية. ع ـ ابنية الجملة المختلفة عن اللغة التي يتقنها المتعلم وغيره.

ستؤدي هذه المشكلات إلى تعطيل عملية التعلم إلى حد كبير، إذا لم يتم إدارها

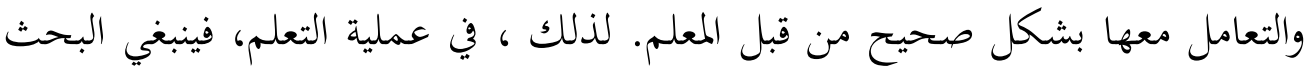
عن طرق وتقنيات توافق احوال المتعلمين و قدرقم. تقنية التعليمية أو الاستراتيجية التي

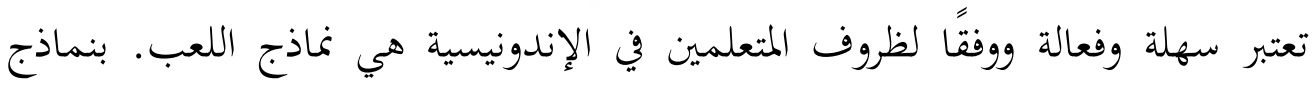
اللعب سيكون الأطفال سعداء بالتعلم، حتى يكون عملية التعلم فعالية وتحتاج إلى

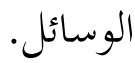

(Umi Machmudah dan Abdul Wahab Rosyidi, 2008, 7.0)

وسائل الإعلام هي توجيه المركبة أو حاوية رسائل التعلمية. وبالتالي، يلعب تعلم

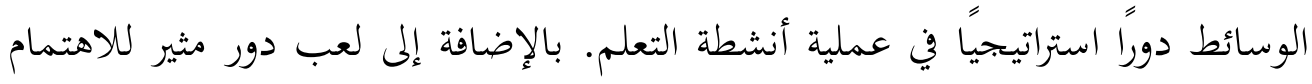
أو اهتمام بالطلاب، يلعب أيضًا دوراً في نقل الرسالة التي يراد إيصالها في كل مادة. لإنشاء جو تعليمي يجذب انتباه الطلاب، يتطلب إنشاء معلم وابتكاره وتنوعه في تصميم بحموعة متنوعة من وسائل التعلم بحيث يمكن إجراء أنشطة التعلم من خحلال تحسين العملية والتوجه نحو تحقيق أو نتائج التعلم.

هناك نظريات مختلفة تتعلق بالتعلم. التعلم هو تغيير في السلوك يحدث من خلال

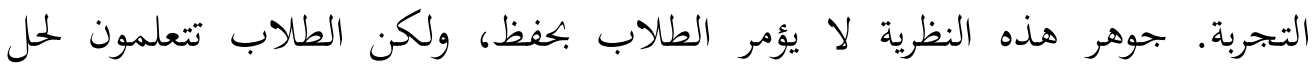
المشاكل، وتشكيل الفرضيات واختبارها. في النهاية، من خلال توجيهات المعلم، يمكن 
للطلاب إجراء استتاجات. تتطلب مثل هذه الاستراتيجيات طلابًا أكثر نشاطًا، بينما يساعد المعلمون في التوجيه فقط.

بتحليل نظرية التعلم أعلاه، فإن تعلم اللغة العربية مناسبة جدًا عند تطبيق ذلك النظرية. في الممارسة العملية، المعلم في هذه الحالة يتطلب وسائل الإعلام التعلمية. كما هو معروف أن وسيلة التعلم هي وسيطة لمصادر الرسائل التي تلعب دورًا مهمًا في عملية

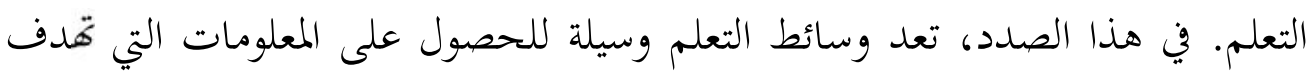
إلى عملية التعلم لدى الطلاب بحيث تكون هناك تغييرات في السلوك، سواء في شكل المعرين (المعرفة)، العاطفي (الموقف) والحركي النفسي (المهارات). في عصر العولمة الحالي، هناك العديد من وسائل الإعلام المختلفة التي تدعم التعلم في المدارس. وسائل الإعلام جاهزة للاستخدام، قد تكون متطورة وحديثة بالفعل تستخدم في المدارس المفضلة. بل يمكن للطلاب الحصول عليها ويبدو أهم يعتمدون بالفعل على وسائل الإعلام. بدون وسائل الإعلام، لا يمكنهم التعلم على أكمل وجحه. علاوة على ذلك، تميل طبيعتها إلى أن تكون فضولية للغاية بشأن الأشياء الجديدة. المشكلة هي كيف يمكن للطلاب في المناطق النائية؟ يمكن أن لديهم وسائل الإعلام الحديثة؟ على الرغم من أهما هي نفسها، كل الفضوليين. إذا كانت الظروف هكذا، يحدد

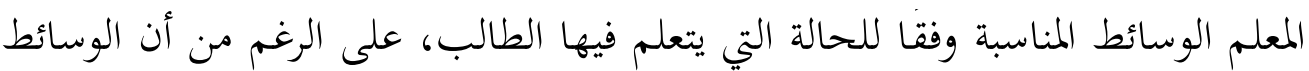
بسيطة أو غير حديثة. الجوهر هو تحقيق هدف التعلم. فيما يتعلق بدور وسائل الإعلام التعليمية، قال الفوزان إن لوسائل الإعلام دوراً

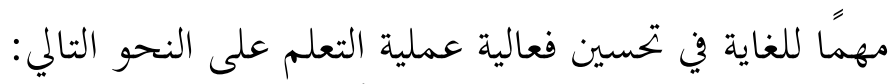

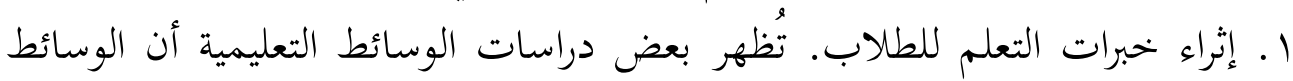
تلعب دورًا مهمًا في إثراء خبرات التعلم لأن الطلاب يشهدون ويشعرون بشكل 
مباشر بموضوع النقاش الذي تمت مناقشته في الفصل ويمكنهم تسهيل فهمه لأنه يتم نقله بطريقة مثيرة للاهتمام من خلال بعض الوسائط.

r. اقتصادية. والمقصود بالاقتصاد هنا هو أن عملية التعليم والتعلم باستخدام الوسائط سوف تكون قادرة على تقديم محاضر التعلم بفعالية في وقت سريع نسبيًا مقارنة دون استخدام الوسائط، وفي نفس الوقت هناك حاجة إلى الطاقة لنقل أو شرح عدد أقل نسبياً من الدروس. r. زيادة اهتمام الطلاب بالدرس. من خلال وسائل الإعلام التعليمية، سيكون الموضوع

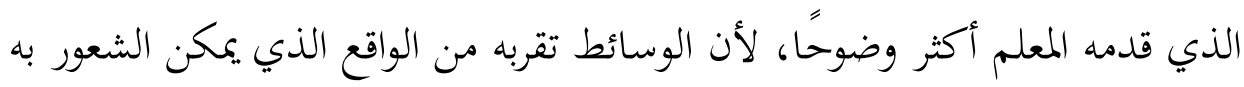

$$
\text { مباشرة. }
$$

ع. اجعل الطلاب أكثر استعدادًا للتعلم. باستخدام وسائط التعلم ، يكصل الطلاب على خبرة عملية، وتكون مواقف التعلم أكثر فاعلية وتؤدي إلى نتائج أفضل. ه. ضم العديد من الحواس الخمس في عملية التعلم. كلما زاد عدد المشاركين من الحواس الخمس المشاركة في عملية التعلم، من المتوقع أن تستمر نتائج الطلاب لفترة أطول حتى تصبح جودة التعلم أفضل. ج. تقليل الاختلافات في التصورات بين المعلمين والطلاب. غالبًا ما تكون هناك تصورات مختلفة في تفسير شيء ما، خاصة في تعلم اللغة، على سبيل المثال lafadz والتفاهم الوثيق بين المعلمين والطلاب، ثم يصبح استخدام وسائط التعلم مهمًا للغاية، لأن الوسائط يمكن أن تغير شيئًا بحردة تصبح شيئا يمكن أن يستشعر. 
V. إضافة مساهمات إيهابية من الطلاب في اكتساب خبرة التعلم. وذلك لأن تعلم الوسائط يمكن أن يطور قدرة الطلاب على التفكير والتحليل لإيجاد استتاجات

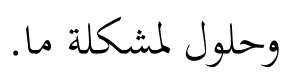
ه. يساعد في حل الاختلافات الشخصية بين الطلاب. كل طالب في الفصل لديه قدرة غير متكافئة، يككن لهذا التنوع في بعض الأحيان أن يخلق مشاكله الخاصة في عملية التعليم والتعلم، على مسبيل المثال لا يزال هناك طلاب لم يتمكنوا من تلقي الدروس

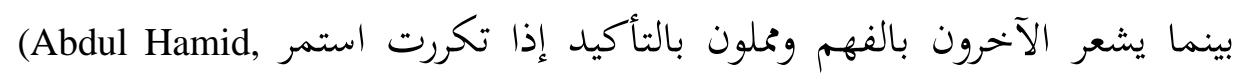
dkk.2008 171-174.)

\section{د. أنواع وسائل الإعلام لتعلم اللغة}

بشكل عام، تنقسم الوسائل في تعلم اللغة إلى ثلاثة أنواع، هي: (1) الوسائل

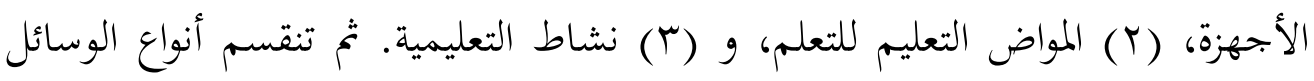

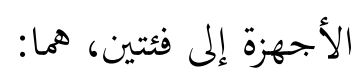
1 ـ الأجهزة الفنية التي تشمل: الأجهزة السمعية مثل أجهزة الراديو ومسجلات الأشرطة والأقراص المدبحة البسيطة والمعامل اللغوية.

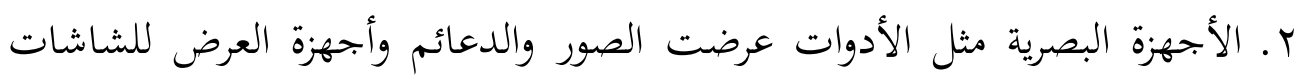

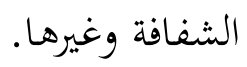


r. الأجهزة السمعية البصرية مثل التلفزيون والفيديو وشاشات الكريستال السائل

$$
\text { وغيرها. }
$$

ع. الأجهزة الإلكترونية مثل أجهزة الكمبيوتر.

فاما وسائل مواد التعليمية والتعلمية تنقسم إلى ثلاث أنواع :

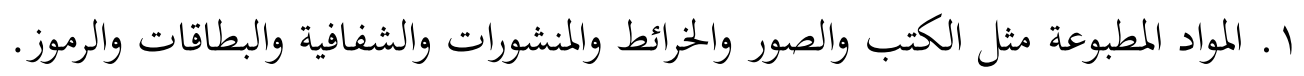

r. المواد السمعية البصرية الثابتة، مثل الأفلام غير الثابتة وما أشبهـها. r. المواد السمعية البصرية المتحركة، مثل الأفلام وأشرطة الفيديو والفيديو.

وأما أنواع الوسائل لأنشطة دعم التعلم (النشاط التعليمية مثل: الأنشطة والزيارات

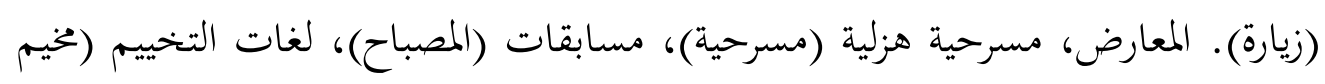
لغاوي).

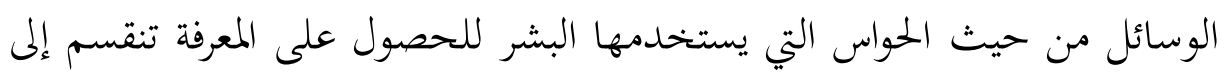

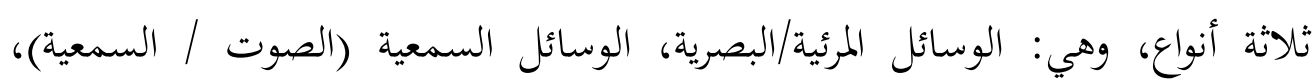

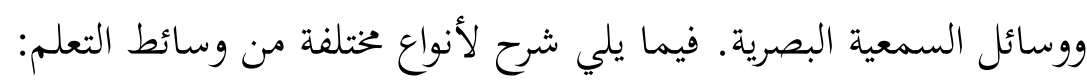

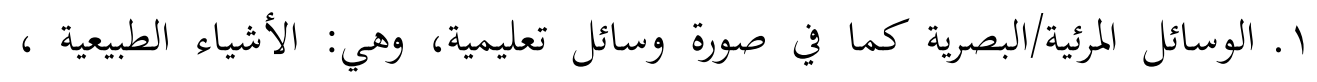
والأشخاص والأحداث، وتقليد الأشياء الطبيعية، وصور الأشياء الطبيعية. r. الوسائل السمعية (الصوت / السمعية) التي يمكن استخدامها لتدريس اللغة وهي

$$
\text { r. الإذاعة ومسجل الشريط ومختبر اللغة (بسيط). }
$$

إن أكثر وسائل تعليمية اللغة اكتمالًا هي الوسائل السمعية البصرية، لأنه في هذه النهابه

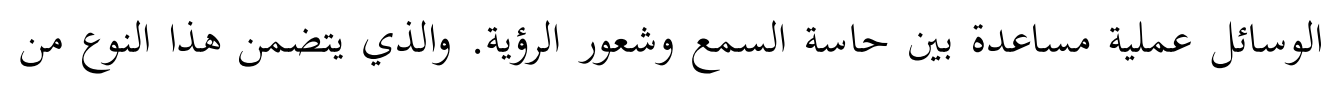


وسائل الإعلام التلفزيونية، VCD، الكمبيوتر وآخر مختبر اللغة. Abdul Hamid,

dkk.,2008 174-175.)

\section{هـ معايير اختيار الوسائل والإجراءات}

لاختيار واستخدام الوسائل الصحيحة في التعلم، يجب على المعلم مراعاة العديد

$$
\text { من الأشياء المتعلقة بمعايير وإجراءات تحديد وسائل التعلمية : }
$$

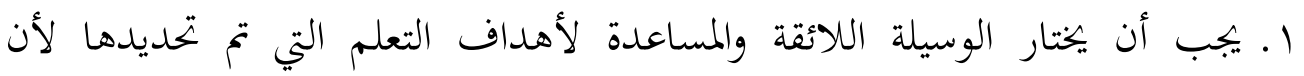

أهداف التعلم هي العنصر المهم يجب مراعاته عند اختيار الوسائل. في تحديد وسائل

الإعلام يجب أن تكون وعملية ومحددة، وينعكس حقا في شكل سلوك (السلوك).

r. تعتبر المادة مهمة في اختيار الوسائل. سيكون الوسائل المستخدمة تأثير على نتائج

$$
\text { تعلم الطلاب. }
$$

r. تصبح حالة الطالب أو موضوع الدراسة مصدر قلق بالغ للمعلم في اختيار الوسيلة المناسبة لحالة الطفل. فلذلك كان العامل العام والذكاء والخلفية التعليمية والثقافة والبيئة للأطفال هي نقاط الاهتمام والنظر في اختيار وسائل الإعلام التعليمية. ع. إن توفير وسائل الإعلام في المدارس يمكن للمعلمين تصميم وسائلهم لاستخدامها

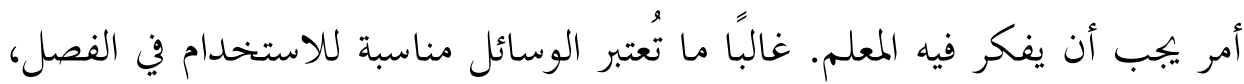
لكن المدرسة لا تملك وسائل الإعلام أو المعدات اللازمات المطلوبات حتى يكون

$$
\text { تصميم الوسائل المطلوبات غير ممكن للمعلم. }
$$

ه. يجب أن تكون الوسيلة المحددة قادرة على شرح ما يراد إلى الطلاب بشكل صحيح وفعال، بعبارة أخرى، يمكن تحقيق الأهداف المحدة على النحو الأمثل. 
7. ينبغي ان تكون موازنة التكاليف تكبد في استخدام الوسائل مماثلة للنتيجة المرادة. قد يكون الاستخدام البسيط للوسائل أكثر ربكية من استخدام الوسائل المتطورة (التكنولوجية العالية) لكن النتائج ليست مماثلة بالأموال التي يتم إنفاقها.

\section{و. مبادئ استخدام وسائل الإعلام في تعليم اللغة العربية}

يتم استخدام الوسائل التعلم في سياق الجهود المبذولة لزيادة أو تحسين جودة عملية

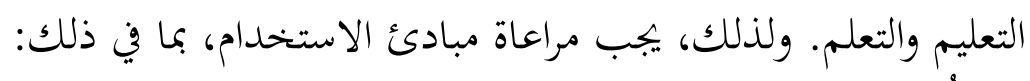

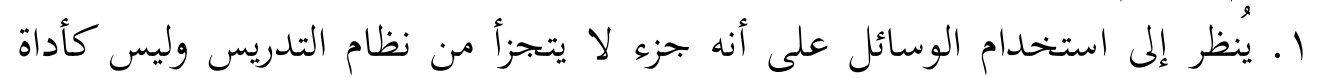
مساعدة عند الضرورة وتستخدم عند الحاجة فقط.

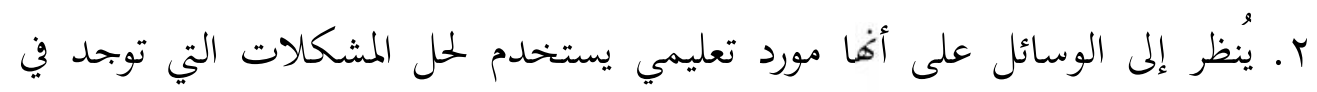
عملية التعليم والتعلم.

r. كان المعلم اهلا في تقنية وسيلة التدريس المستخدمة. ع. يأخذ المعلم في الاعتبار ربحا أو خسارة في استخدام الوسائل. ه. يجب تنظيم استخدام الوسائل بشكل منهجي بدلاً من استخدامها فقط.

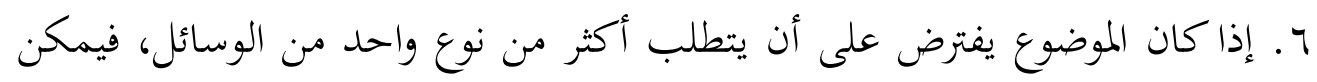
للمعلم استخدام الوسائل المتعددات التي تستفيد وتسهل عملية التدريس والتعلم

$$
\text { ويمكنها أيضًا تحفيز الطلاب على التعلم. }
$$

بعض المتطلبات العامة التي يجب الوفاء هما عند استخدام الوسائل في عملية التعليم

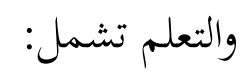

1. يجب أن تكون الوسيلة المستخدمة وفقًا لأهداف التعلم المقصودة. 
r. الوسائل هي وسيلة يككن رؤيتها أو سماعها. r. وسائل الإعلام المستخدمة يمكن أن تستجيب لتعلم الطلاب.

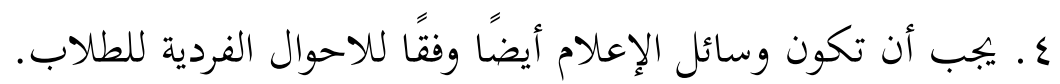
ه. الوسائل هي وسيط (متوسط) في عملية تعلم الطلاب.

يجب أن يأخذ استخدام الوسيلة التعليمية في الاعتبار ما يلي:

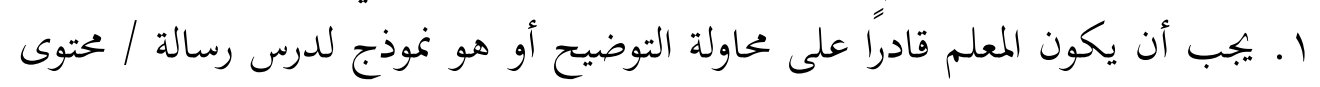

\section{تم تسليمه.}

r. إذا لم يكن بالإمكان إحضار الكائن المطلوب عرضه في الفصل، جاء طلاب الفصل

$$
\text { لتحديد موقع الكائن. }
$$

r. إذا لم يمكن ان بتجئ طلاب الفصل إلى موقع الكائن ، فجرب النموذج أو التقليد.

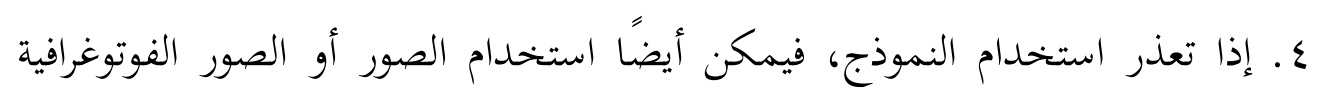

$$
\text { للأشياء التي ترضي الموضوع. }
$$

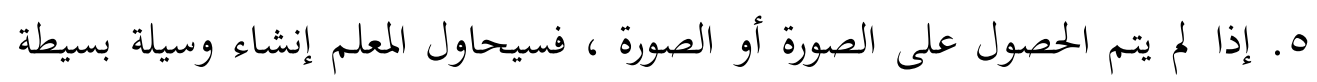

$$
\text { يمكنها جذب انتباه الطلاب. }
$$

I. عندما يتعذر إنشاء وسيلة بسيطة، يستخدم المعلم السبورة لتوضيح الكائن أو الرسالة

$$
\text { من خلال صورة بسيطة. }
$$

من العبارة الماضية يعرف انه لم يعد اعتماد العملية التعليمية على الوسائل مظهرا

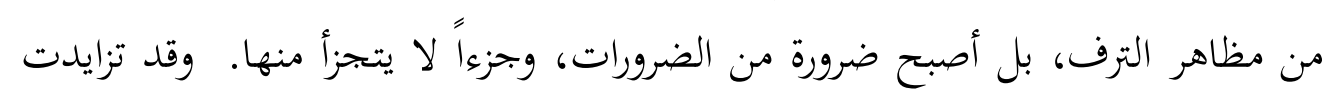

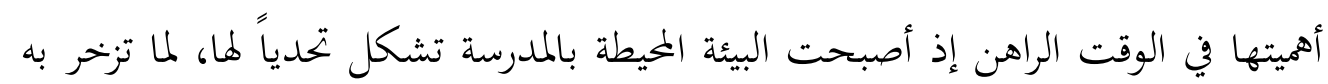
هذه البيئة من وسائل اتصال متنوعة تتسم بالجذب والإثارة، وحتى لا تفقد المدرسة دورها 
في التعليم والتربية كان لا بدّ لها من تحديث أنشطتها التعليمية والتربوية، وفي مقدمة ذلك الوسائل التعليمية.(Kisbiyanto, 313)

\section{ز. الغرض من استخدام وسائل الإعلام في تعلم وتعليم اللغة العربية}

يماول معلمو اللغة العربية في أداء واجباتم في الفصل الدراسي القيام بالتعلم

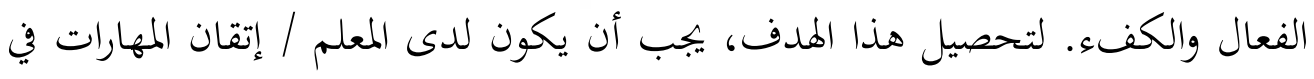
استخدام وسائل التعلم.

كما أوضح يوسف ميارسو، فإن المعلم الذي يستخدم الوسائل لمصدر التعلم 1 يهدف تطوير التعلميم التكنولوجي / المندسم التصرف بشكل احترافي في الأمور كما يلي: r. تصميم وتطوير عمليات التعلم والمصادر والأنظمة.

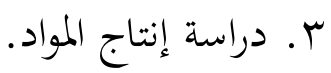
ع ـ توفير مرافق التعلم والبنية التحتية. 0. اختيار نظم وتقييمه ومكونات نظام التعلم. 7. الاستفادة من عملية التعلم ومصادره. V V V لشر مفاهيم / نتائج التكنولوجيا / وسائل التعلم. 1. إدارة أنشطة تطوير واستخدام مصادر التعلم.

9. صياغة مواد للتكنولوجيا / هندسة التعلم. Yusufhadi, Miarso, 2004, 70.) في شرح أكثر تفصيلاً، أوضح يوسف هدي ميارسو أن المعلم في المرحلة الجامعية الأولى يجب أن يتمتع بالمهارات في استخدام الوسائل مع إتقان القدرات التالية: 


$$
\begin{aligned}
& \text { 1. فهم الأسس النظرية / بحث وتطبيق التكنولوجيا التعليمية. } \\
& \text { r - تصميم أنماط تعليمية. }
\end{aligned}
$$

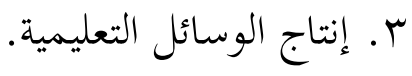

$$
\begin{aligned}
& \text { ع. تقييم البرامج التعليمية والإنتاج. } \\
& \text { ه. إدارة وسائل الإعلام وأدوات التعلم. } \\
& \text { 7. الاستفادة من المرافق ووسائل الإعلام والتقنيات التعليمية. } \\
& \text { V. نشر منتجات التكنولوجيا والمعلومات التعليمية. }
\end{aligned}
$$

م. تعمل وحدها وتدريب الآخرين في تشغيل المعدات السمعية والبصرية. Yusufhadi, Miarso, 91.)

من العبارة أعلاه، يفهم أن استخدام الوسائل في تعلمية اللغة العربية يهدف إلى ما

$$
\text { ا. مع وسائل الإعلام، يمكن لمدرسي اللغة العربية تحقيق التعلم بكفاءة وفعالية. }
$$

r. من خلال استخدام وسائل الإعلام، يزداد المعلمون مهارة في تخطيط عملية التعلم

$$
\text { وتقييمها. }
$$

r. باستخدام الوسائل، يمكن لمدرسي اللغة العربية إنشاء وسيلة / مصدر جديدة في

$$
\text { التعلم بعد استخدام بحموعة متنوعة من الوسائط السابقة. }
$$

ع. من خلال استخدام الوسائل في كثير من الأحيان، يمكن لمدرسي اللغة العربية نشر

$$
\text { نتائج أحدث الابتكارات في التعلم. }
$$


هـ باستخدام الوسائل، يمكن لمدرسي اللغة العربية تطوير جودة التعليم والتدريس في الفصل الدراسي مع المزيد من التخطيط والتكامل والإبداع، حتى اكتشاف نماذج جديدة للتعلم من بين المعلمين أنفسهم.

\section{ح. يجب أن يكون معلمو اللغة العربية مؤهلين في استخدام وسائل الإعلام}

لتحقيق التعلم الفعال، أن للمعلم دور كبير. المعلمون الفعالون هم المعلمون الذين

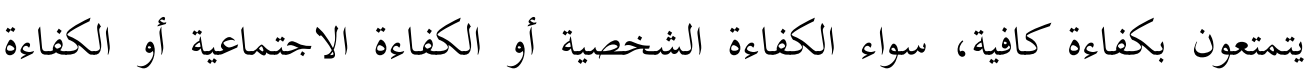
المهنية. يتم دعم الكفاءة المهنية للمعلمين من خلال تعليم المعلمين، لأن التحيز في تعليم المعلمين يمكن أن يكون له القدرة على إتقان مواد التدريس، والقدرة على تدريسها والقدرة على تطوير التعلم. واحدة من الكفاءات التي يجب أن يمتلكها المعلم هي القدرة على استخدام الوسائل في التعلم.

تتأثر الكفاءة المهنية للمعلمين بالعديد من الكفاءات الفرعية، بما في ذلك القدرة على استخدام الوسائط. يعني أن معلمي اللغة العربية المحترفين هم المدرسون لديهم القدرة على فهم الأساس النظري/البحث وتطبيق التكنولوجيا التعليمية، وتقييم البرامج والمنتجات التعليمية، وإدارة وسائل الإعلام والتعلم، والاستفادة من المرافق ووسائل الإعلام والتقنيات التعليمية، ونشر منتجات تكنولوجيا المعلومات والتعليم، تعمل وحدها وتدريب الآخرين في تشغيل المعدات السمعية والبصرية.(Kisbiyanto, 315) 


\section{الخحلاصة}

من الأمور السابقة يمكن الاستنباط :

ا ـ تعد الوسائل التعليمة أحد العوامل الحاسمة للغاية في دعم بجاح عملية التعلم في

$$
\text { الفصل الدراسي وتحقيق الأهداف التعليمية. }
$$

r. تعتبر الوسائل التعليمة بديلاً للمعلمين في جلب الطلاب لفهم المواد التعليمية

$$
\text { المقدمة بسهولة }
$$

ب. الوسائل التعليمية للّغة العربية هي مجموعة من أدوات التعلم التي أعدها المعلم لشرح الرسائل ذات الصلة بالمواد والأدوات التعليمية التي بجذب الانتباه واهتمام الطلاب بالتعلم حول المواد العربية. فيما يتعلق بالتنوع والوظائف في مختلف وسائل التعلم، يجب اختيار وسائط التعلم وتكييفها مع أهداف التعلم المراد تحقيقها، ومدى ملاعمة حالة الطالب، ومدى توافرها، والجودة، والتقنية، والتكلفة

$$
\text { والمزيد من الاهتمام من قبل مدرس اللغة العربية. }
$$




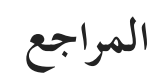

Abdul Hamid, dkk., Pembelajaran Bahasa Arab (Pendekatan, Metode, Strategi, Materi, dan Media), Yogyakarta: UIN Malang Press, 2008.

Azhar Arsyad, Media Pembelajaran, Jakarta: PT. Raja Grafindo Persada, 2007.

Djudju Sudjana S, Strategi Pembelajaran, Bandung: Falah Production, 2000.

Kisbiyanto, Media Pembelajaran Bahas Arab, Jurnal Pendidikan Bahasa Arab Jurusan Tarbiyah STAIN KUDUS Vol. 3 No. 2, Juli-Desember 2011.

Soenjoyo Dirjo Soemarto, Dalam Azhar Arsyad, Bahasa Arab dan Metode Pengajarannya: Beberapa Pokok Pikiran, Yogyakarta: Pustaka Pelajar, 2003.

Umi Machmudah dan Abdul Wahab Rosyidi, Active Learning dalam Pembelajaran Bahasa Arab, Malang: UIN Malang Press, 2008.

Yusufhadi, Miarso, Menyemai Benih Teknologi Pendidikan, Jakarta: Prenada Media, 2004. 